\title{
A Note of Filters in Effect Algebras
}

\author{
Biao Long Meng ${ }^{1}$ and Xiao Long Xin ${ }^{2}$ \\ ${ }^{1}$ Department of Mathematics, Xian University of Science and Technology, Xian 710054, China \\ ${ }^{2}$ Department of Mathematics, Northwest University, Xian 710127, China
}

Correspondence should be addressed to Xiao Long Xin; xlxin@nwu.edu.cn

Received 29 July 2013; Accepted 23 September 2013

Academic Editors: Z.-Y. Li and C.-s. Liu

Copyright (C) 2013 B. L. Meng and X. L. Xin. This is an open access article distributed under the Creative Commons Attribution License, which permits unrestricted use, distribution, and reproduction in any medium, provided the original work is properly cited.

We investigate relations of the two classes of filters in effect algebras (resp., MV-algebras). We prove that a lattice filter in a lattice ordered effect algebra (resp., MV-algebra) $E$ does not need to be an effect algebra filter (resp., MV-filter). In general, in MV-algebras, every MV-filter is also a lattice filter. Every lattice filter in a lattice ordered effect algebra $E$ is an effect algebra filter if and only if $E$ is an orthomodular lattice. Every lattice filter in an MV-algebra $E$ is an MV-filter if and only if $E$ is a Boolean algebra.

\section{Introduction}

The notion of effect algebras has been introduced by Foulis and Bennett [1] as an algebraic structure providing an instrument for studying quantum effects that may be unsharp. One of D-posets has been introduced by Chovanec and Kôpka [2]. The two notions are categorically equivalent. Many results with respect to effect algebras and D-posets have been obtained (see [1-6]). A comprehensive introduction about effect algebras can been found in the monograph [7]. The filter theory of effect algebras is an important objects of investigation (see $[3,4,8,9]$ ). It is well known that a lattice ordered effect algebra contains both a lattice structure and an effect algebra structure; hence, the notions of lattice filters and effect algebra filters are investigated, respectively. One would ask: what relations are there between lattice filters and effect algebra filters? In this paper we discuss this problem in a lattice ordered effect algebra $E$ (resp., an MV-algebra). A lattice filter in a lattice ordered effect algebra does not need to be an effect algebra filter (resp., MV-filter). In general, lattice filter in a lattice ordered effect algebra $E$ is an effect algebra filter if and only if $E$ is an orthomodular lattice. A lattice filter in an MV-algebra $E$ is an MV-filter if and only if $E$ is a Boolean algebra.

Definition 1 (see [1]). An effect algebra is an algebraic structure $(E ; \oplus, 0,1)$, where $E$ is a nonempty set, 0 and 1 are distinct elements of $E$, and $\oplus$ is a partial binary operation on $E$ that satisfies the following conditions.

(E1) Commutative Law. If $a \oplus b$ is defined, then $b \oplus a$ is defined and $a \oplus b=b \oplus a$.

(E2) Associative Law. If $a \oplus b$ and $(a \oplus b) \oplus c$ are defined, then $b \oplus c$ and $a \oplus(b \oplus c)$ are defined and $(a \oplus b) \oplus c$ $=a \oplus(b \oplus c)$.

(E3) Orthosupplementation Law. For any $a \in E$, there is a unique $a^{\prime} \in E$, such that $a \oplus a^{\prime}=1$.

(E4) Zero-One Law. If $a \oplus 1$ is defined, then $a=0$.

When the hypotheses of (E2) are satisfied, we write $a \oplus b \oplus c$ for element $(a \oplus b) \oplus c=a \oplus(b \oplus c)$ in $E$.

For simplicity, we use the notation $E$ for an effect algebra. If $a \oplus b$ is defined, we write $a \perp b$ and whenever we write $a \oplus b$ we are implicitly assuming that $a \perp b$. A partial ordering on an effect algebra is defined by $a \leq b$ if and only if there is a $c \in E$, such that $a \oplus c=b$. Such an element $c$ is unique (if it exists) and is denoted by $b \ominus a$. Then $(E ; \ominus, 0,1)$ is a difference poset (D-poset, for short) [2]. In this case we note $a^{\prime}:=1 \ominus a$. It is known that for any $a \in E, a^{\prime \prime}:=\left(a^{\prime}\right)^{\prime}=a$ and $a \leq b$ implies $b^{\prime} \leq a^{\prime}$.

Definition 2 (see [4]). An orthoalgebra is an algebraic structure $(E ; \oplus, 0,1)$ satisfies $(\mathrm{E} 1)-(\mathrm{E} 3)$ and the following condition. 
Consistency Law. If $a \oplus a$ is defined, then $a=0$.

An orthoalgebra is always an effect algebra. An effect algebra (orthoalgebra, resp.) with lattice order is called a lattice ordered effect algebra (lattice ordered orthoalgebra, resp.).

Let $E$ be an orthoalgebra. If $a, b \in E$ and there are 1.u.d $\{a, b\}$ and g.l.d $\{a, b\}$, then we denote $a \vee b:=1 . u . d\{a, b\}$ and $a \wedge b:=$ g.l.d $\{a, b\}$. Then the map ' ${ }^{\prime} a \mapsto a^{\prime}$ is an orthocomplementation on the bounded poset $(E ; \leq)$, that is, the following conditions hold: for all $a, b \in E$,

$$
\begin{aligned}
& \text { (1) } a^{\prime \prime}:=\left(a^{\prime}\right)^{\prime}=a, \\
& \text { (2) } a \leq b \Rightarrow b^{\prime} \leq a^{\prime}, \\
& \text { (3) } a \wedge a^{\prime}=0, \\
& \text { (4) } a \vee a^{\prime}=1 .
\end{aligned}
$$

Definition 3 (see [5]). An orthocomplementation lattice $\left(E ; \vee, \wedge,{ }^{\prime}, 0,1\right)$ is called an orthomodular lattice if it satisfies the orthomodular Law:

$$
\begin{aligned}
& \text { for any } a, b \in E, a \leq b \text { implies } b=a \vee\left(a \vee b^{\prime}\right)^{\prime} \\
& \quad \text { or equivalently } b=a \vee(b \ominus a) .
\end{aligned}
$$

Lemma 4. In an effect algebra $E, a \perp b$ if and only if $a \leq b^{\prime}$.

Lemma 5. A lattice ordered orthoalgebra is just an orthomodular lattice.

Hence, in an orthomudular lattice, the complementation operation in a lattice is the same as the orthosupplement operation in an effect algebra.

\section{Filters in Lattice Effect Algebras}

Definition 6 (see [3]). Let $(E ; \oplus, 0,1)$ be an effect algebra. In the terms of the effect algebra operation $\oplus$, a partial operation $\odot$ can be defined as follows: for any $a, b \in E$

$a \odot b$ exists if and only if $a^{\prime} \perp b^{\prime}, a \odot b={ }_{\mathrm{def}}\left(a^{\prime} \oplus b^{\prime}\right)^{\prime}$.

The following assertions are obvious and the proofs are omitted.

Proposition 7. Let $E$ be an effect algebra. Then for any $a, b, c \in$ E:

(i) If $a \odot b$ exists, then $b \odot a$ exists and $a \odot b=b \odot a$.

(ii) If $b \odot c$ and $a \odot(b \odot c)$ exist, then $a \odot b$ and $(a \odot b) \odot c$ exist and $a \odot(b \odot c)=(a \odot b) \odot c$.

(iii) For any $a$ there is $a b$, such that $a \odot b=0$ and $b=a^{\prime}$.

(iv) If $0 \odot a$ exists, then $a=1$.

(v) For any $a \in E, a \odot a^{\prime}$ exists and $a \odot a^{\prime}=0$.

(vi) $a \odot b$ exists if and only if $a^{\prime} \leq b$.

(vii) If $E$ is a lattice ordered effect algebra, then for all $a, b \in$ $E$ with $a^{\prime} \perp b^{\prime}$, we have $a \odot b \leq a \wedge b$.
Definition 8 (see [9]). Let $(E ; \oplus, 0,1)$ be an effect algebra and $F$ be a nonempty subset of $E$. Then $F$ is called an effect algebra filter on $E$ if for all $a, b \in E$ with $a^{\prime} \perp b^{\prime}, a, b \in F$ if and only if $a \odot b \in F$. An effect algebra filter $F$ of $E$ is called to be proper if $F \neq E$.

Obviously, an effect algebra filter $F$ of $E$ is proper if and only if $0 \notin F$.

Definition 9 (see [4]). Let $(L ; \vee, \wedge, 0,1)$ be a lattice. A nonempty subset $F$ of $L$ is called a lattice filter of $L$ if for all $a, b \in L, a, b \in F$ if and only if $a \wedge b \in F$.

Proposition 10. Let $(E ; \oplus, 0,1)$ be an effect algebra. A nonempty subset $F$ of $E$ is an effect algebra filter of $E$ if and only if $F$ satisfies:

$\left(E F_{1}\right)$ if $a \in F$ and $a \leq b$, then $b \in F$.

$\left(E F_{2}\right)$ For $a, b \in E$ with $b \leq a$, then $(a \ominus b)^{\prime} \in F$ and $a \in F$ imply $b \in F$.

Proof. Let $F$ be an effect algebra filter of $E$. If $a \in F$ and $a \leq b$, then $\left(a^{\prime} \ominus b^{\prime}\right)^{\prime} \odot b=\left(\left(a^{\prime} \ominus b^{\prime}\right) \oplus b^{\prime}\right)^{\prime}=a \in F$. So $b \in F$. $\left(\mathrm{EF}_{1}\right)$ holds. Suppose that $b \leq a$ and $(a \ominus b)^{\prime} \in F, a \in F$. Then $\left(b^{\prime} \ominus a^{\prime}\right)^{\prime} \in F$, and $b=\left(\left(b^{\prime} \ominus a^{\prime}\right) \oplus a^{\prime}\right)^{\prime}=(a \ominus b)^{\prime} \odot a \in F$. $\left(\mathrm{EF}_{2}\right)$ holds.

Conversely suppose $F$ satisfies $\left(\mathrm{EF}_{1}\right)$ and $\left(\mathrm{EF}_{2}\right)$. Let $a, b \in$ $F$ with $a^{\prime} \perp b^{\prime}$. Since $(b \ominus(a \odot b))^{\prime}=\left((a \odot b)^{\prime} \ominus b^{\prime}\right)^{\prime}=$ $\left(\left(a^{\prime} \oplus b^{\prime}\right) \ominus b^{\prime}\right)^{\prime}=a \in F$ and $b \in F$, by $\left(\mathrm{EF}_{2}\right)$ we obtain $a \odot b \in I$. On the other hand, if $a, b \in E$ with $a^{\prime} \perp b^{\prime}$ and $a \odot b \in F$, then by $a \odot b \leq a, b$ and $\left(\mathrm{EF}_{1}\right)$ we have $a, b \in F$. Hence, $F$ be an effect algebra filter of $E$.

Proposition 11. Let $(L ; \vee, \wedge, 0,1)$ be a lattice. A nonempty subset $F$ of $L$ is a lattice filter of $L$ if and only if $F$ satisfies the following.

$\left(L F_{1}\right)$ If $a \in F$ and $a \leq b$, then $b \in F$.

$\left(L F_{2}\right)$ If $a, b \in F$, then $a \wedge b \in F$.

Proof. Let $F$ be a lattice filter of $L$. If $a \in F$ and $a \leq b$, then $a \wedge b=a \in F$. So $b \in I,\left(\mathrm{LF}_{1}\right)$ holds. $\left(\mathrm{LF}_{2}\right)$ holds obviously.

Conversely suppose $F$ satisfies $\left(\mathrm{LF}_{1}\right)$ and $\left(\mathrm{LF}_{2}\right)$. Let $a, b \in$ $F$. By $\left(\mathrm{LF}_{2}\right)$ we obtain $a \wedge b \in F$. On the other hand, if $a, b \in E$ with $a \wedge b \in F$, then by $a \wedge b \leq a, b$ and $\left(\mathrm{LF}_{1}\right)$ we have $a, b \in F$. Hence, $F$ is a lattice filter of $L$.

It is worth noting that in a lattice ordered effect algebra, a lattice filter does not need to be an effect algebra filter.

Example 12. Let $E=\{0, a, b, 1\}$ with $a \neq b, a=a^{\prime}, b=b^{\prime}$, $1=a \oplus a=b \oplus b=a \vee b$, and $a \not \supset b, x \oplus 0=x(x \in E)$. The order relations are as the following picture

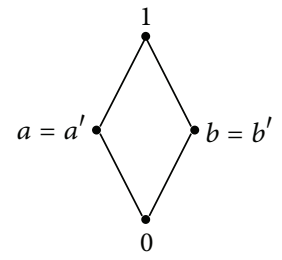


Then $(E ; \oplus, 0,1)$ is a lattice ordered effect algebra. $F=$ $\{a, 1\}$ is a lattice filter of $E$, but $F$ is not an effect algebra filter of $E$ because $a \in F, a \odot a=(a \oplus a)^{\prime}=1^{\prime}=0 \notin F$.

By the way we point out that in the example, the orthosupplement of $a$ in the effect algebra is $a$ and the orthocomplement of $a$ in the lattice is $b$; they are different.

In order to make a lattice filter of $E$ also being an effect algebra filter of $E$, we must add stronger conditions on $E$.

Theorem 13. Let $(E ; \oplus, 0,1)$ be a lattice ordered effect algebra. Then the following conditions are equivalent.

(a) Every lattice filter is an effect algebra filter.

(b) For all $a, b \in E$ with $a^{\prime} \perp b^{\prime}, a \wedge b=a \odot b$.

(c) $E$ is a lattice ordered orthoalgebra.

Proof. (a) $\Rightarrow$ (b). Assume (a) and $a, b \in E$ with $a^{\prime} \perp b^{\prime}$. Since $[a \wedge b, 1]$ is a lattice filter of $E$ and $a, b \in[a \wedge b, 1]$, by the assumption $[a \wedge b, 1]$ is also an effect algebra filter of $E$, it follows that $a \odot b \in[a \wedge b, 1]$, and so $a \wedge b \leq a \odot b$. Thus, $a \odot b=a \wedge b$. (b) holds.

(b) $\Rightarrow$ (c). Assume (b) and $a$ is any element of $E$. By (b) and Proposition 7(v), we have $a \wedge a^{\prime}=a \odot a^{\prime}=0$. By Part (iii) of Proposition 1.5.3 in [7], $E$ is an orthoalgebra. (c) holds.

(c) $\Rightarrow$ (a) Suppose $E$ is an orthoalgebra and $F$ is any lattice filter of $E$. If $a, b \in F$ with $a^{\prime} \perp b^{\prime}$, then $a \wedge b \in F$. Because $(a \wedge b)^{\prime}=a^{\prime} \vee b^{\prime} \leq a^{\prime} \oplus b^{\prime}=(a \odot b)^{\prime}$, it follows from Part (ii) of Proposition 1.5.3 in [7] that $(a \wedge b)^{\prime}=(a \odot b)^{\prime}$, and so $a \wedge b=a \odot b$. Therefore, $a \odot b \in F$. Conversely if $a \odot b \in F$, by $a \odot b \leq a, b$ and $\left(\operatorname{LF}_{1}\right)$, we have $a, b \in F$. This prove that $F$ is an effect algebra filter of $E$. (a) holds.

By Lemma 5 and Theorem 13 we have the following.

Corollary 14. Let $E$ be a lattice ordered effect algebra. Then every lattice filtar $F$ is an effect algebra filter if and only if $E$ is an orthomodular lattice.

\section{Filters in MV-Algebras}

In 1959, Chang [10] introduced MV-algebras, which play an important role in many valued logic. An MV-algebra is a very important special example of an effect algebra (equivalently, D-poset). An algebraic structure $\left(E ;+,{ }^{\prime}, 0,1\right)$ is called an $M V$-algebra if $E$ is a nonempty set, 0 and 1 are distinct elements of $E$, + is a total binary operation on $E$, and ' is a unary operation on $E$ satisfying

(MV1) $(a+b)+c=a \oplus(b+c)$

(MV2) $a+0=a$

(MV3) $a+b=b+a$,

(MV4) $a+1=1$,

(MV5) $a^{\prime \prime}:=\left(a^{\prime}\right)^{\prime}=a$,

(MV6) $0^{\prime}=1$,
(MV7) $a+a^{\prime}=1$,

(MV8) $a+\left(a+b^{\prime}\right)^{\prime}=b+\left(b+a^{\prime}\right)^{\prime}$.

In an MV-algebra, we can define total operations $\cdot,-, \mathrm{V}$, and $\wedge$ as follows: for any $a, b \in E, a \cdot b:=\left(a^{\prime}+b^{\prime}\right)^{\prime} ; a \wedge b:=$ $\left(a+b^{\prime}\right) \cdot b ; a \vee b:=\left(a \cdot b^{\prime}\right)+b ; b-a:=\left(a+b^{\prime}\right)^{\prime} ; a \leq b$ if and only if $a=a \wedge b$. Thus, $(E ; \leq,-, 0,1)$ is a Boolean D-poset ([7, Theorem 1.8.16]). An MV-algebra is a bounded distributive lattice with respect to $\vee$ and $\wedge$. Hence, Definition 8 still applies to MV-algebras. The details on MV-algebras can be found in [11]. It is easy to prove the following.

In an MV-algebra $E, a-b=a-(a \wedge b)$ for any $a, b \in E$.

Definition 15. Let $(E ;+, 0,1)$ be an $M V$-algebra and $F$ be a nonempty subset of $E$. Then $F$ is called an MV-filter on $E$ if for all $a, b \in E, a, b \in F$ if and only if $a \cdot b \in F$.

Proposition 16. Let $(E ;+, 0,1)$ be an $M V$-algebra. A nonempty subset $F$ of $E$ is an $M V$-filter of $E$ if and only if $F$ satisfies

$\left(M F_{1}\right)$ if $a \in F$ and $a \leq b$, then $b \in F$.

$\left(M F_{2}\right)$ For $a, b \in E$, then $(a-b)^{\prime} \in F$ and $a \in F$ imply $b \in F$.

Proof. It is analogous to Proposition 10 and omitted.

Observe that in Definition 15 (resp., in Proposition 16), we do not require $a^{\prime} \perp b^{\prime}$.

Proposition 17. Let $(E ;+, 0,1)$ be an $M V$-algebra. If $F$ is an $M V$-filter of $E$, then $F$ is also a lattice filter of $E$.

Proof. Suppose $F$ is an MV-filter of $E$. It follows from Proposition 16 that $F$ satisfies $\left(\operatorname{LF}_{1}\right)$. For any $a, b \in E$ we have

$$
a=(1-a)^{\prime} \leq(b-a)^{\prime}=(b \ominus(a \wedge b))^{\prime} .
$$

Let $a, b \in F$. Then $(b-(a \wedge b))^{\prime} \in F$ by $\left(\mathrm{MF}_{1}\right)$. By using $\left(\mathrm{MF}_{2}\right)$ and $b \in F$, we obtain $a \wedge b \in F$. $\left(\mathrm{LF}_{2}\right)$ holds. From Proposition 11 it follows that $F$ is a lattice filter of $E$.

Even in an MV-algebra, a lattice filter does not need to be an MV-filter.

Example 18. Let $E=\{0, a, b, 1\}$. The Hasse diagram, the tables of operations - and + are as follows:

\begin{tabular}{|c|c|c|c|c|c|c|c|c|c|c|}
\hline & - & 0 & $a$ & $b$ & 1 & + & 0 & $a$ & $b$ & 1 \\
\hline & 0 & 0 & 0 & 0 & 0 & 0 & 0 & $a$ & $b$ & 1 \\
\hline$b$ & $a$ & $a$ & 0 & 0 & 0 & $a$ & $a$ & $b$ & 1 & 1 \\
\hline$a$. & $b$ & $b$ & $a$ & 0 & 0 & $b$ & $b$ & 1 & 1 & 1 \\
\hline 0 . & 1 & 1 & $b$ & $a$ & 0 & 1 & 1 & 1 & 1 & 1 \\
\hline
\end{tabular}

It is easy to check that $E$ is an MV-algebra and $F=\{b, 1\}$ is a lattice filter of $E$, but $F$ is not an effect algebra filter of $E$ because $(b-a)^{\prime}=a^{\prime}=b \in F$ and $b \in F$, while $a \in F$. That is, $F$ does not satisfy $\left(\mathrm{MF}_{2}\right)$.

In what follows we give an interesting result, which is another main conclusion in this paper. 
Proposition 19. Let $(E ;+, 0,1)$ be an $M V$-algebra. Then the following assertions are equivalent.

(i) Every lattice filter of $E$ is an $M V$-filter of $E$.

(ii) For any $a, a \odot a^{\prime}=a \wedge a^{\prime}$.

(iii) $E$ is a Boolean algebra.

Proof. (i) $\Rightarrow$ (ii). Assume (i) and $a \in E$. Since $F:=\left[a \wedge a^{\prime}, 1\right]$ is a lattice filter of $E$ and $a \wedge a^{\prime} \leq a, a^{\prime}$, by $\left(\mathrm{MF}_{2}\right)$ we have $a \odot a^{\prime} \in F$. Hence, $a \wedge a^{\prime} \leq a \odot a^{\prime}$. (ii) holds.

(ii) $\Rightarrow$ (iii). Assume (ii) and $a \in E$. Then $a \vee a^{\prime}=$ $\left(a^{\prime} \wedge a\right)^{\prime}=\left(a \odot a^{\prime}\right)^{\prime}=a+a^{\prime}=1$ by De Morgan Law and (MV8). $E$ is a complemented distributive lattice, that is, a Boolean algebra. (iii) holds.

(iii) $\Rightarrow$ (i). Suppose that $E$ is a Boolean algebra and $F$ is any lattice filter of $E$. It is obvious that $F$ satisfies $\left(\mathrm{MF}_{1}\right)$. Now let $(a-b)^{\prime} \in F$ and $a \in F$, by $\left(\operatorname{LF}_{2}\right)(a-b)^{\prime} \wedge a \in F$. Because

$$
\begin{aligned}
(a-b)^{\prime} \wedge a & =\left((a-b) \vee a^{\prime}\right)^{\prime} \\
& \leq\left((a-b) \vee\left(a^{\prime}-b\right)\right)^{\prime} \\
& =\left(\left(a \vee a^{\prime}\right)-b\right)^{\prime} \\
& =(1-b)^{\prime}=b,
\end{aligned}
$$

by $\left(\mathrm{MF}_{1}\right)$ we have $b \in F$. $F$ satisfies $\left(\mathrm{MF}_{2}\right)$. This shows that $F$ is an MV-filter filter of $E$. (i) holds.

\section{Conclusion}

Ideals and filters play prominent roles in the study of effect algebras. There are various notions of ideals and filters in great literature on effect algebras. The relations among these notions are extensively investigated. It is known that in an effect algebra, lattice filters and effect algebra filters are two important notions. In this paper we show that a lattice filter in a lattice ordered effect algebra is not an effect algebra filter (resp., MV-filter). In general, a lattice filter in a lattice ordered effect algebra $E$ is an effect algebra filter if and only if $E$ is an orthomodular lattice. A lattice filter in an MV-algebra $E$ is an $M V$-filter if and only if $E$ is a Boolean algebra. We will deeply work in this aspect.

\section{Conflict of Interests}

The authors declare that there is no conflict of interests regarding the publication of this paper.

\section{References}

[1] D. J. Foulis and M. K. Bennett, "Effect algebras and unsharp quantum logics," Foundations of Physics, vol. 24, no. 10, pp. 13311352, 1994.

[2] F. Chovanec and F. Kôpka, "D-lattices," International Journal of Theoretical Physics, vol. 34, no. 8, pp. 1297-1302, 1995.

[3] F. Chovanec and E. Rybáriková, "Ideals and filters in D-posets," International Journal of Theoretical Physics, vol. 37, no. 1, pp. 1722, 1998.
[4] D. J. Foulis, R. J. Greechie, and G. T. Rüttimann, "Filters and supports in orthoalgebras," International Journal of Theoretical Physics, vol. 31, no. 5, pp. 789-807, 1992.

[5] G. Jenča and S. Pulmannová, "Ideals and quotients in lattice ordered effect algebras," Soft Computing, vol. 5, no. 5, pp. 376$380,2001$.

[6] Z. Ma, "Note on ideals of effect algebras," Information Sciences, vol. 179, no. 5, pp. 505-507, 2009.

[7] A. Dvurečenskij and S. Pulmannová, New Trends in Quantum Structures, vol. 516 of Mathematics and Its Applications, Kluwer Academic Publishers, Dordrecht, The Netherlands, 2000.

[8] D. L. Liu and G. J. Wang, "Fuzzy filters in effect algebras," Fuzzy Systems and Mathematics, vol. 23, no. 3, pp. 6-16, 2009.

[9] L. Wang and X. N. Zhou, "Prime filters, congruences and quotients in effect algebras," Mathematical Theory and Applications, vol. 27, no. 1, pp. 78-81, 2007.

[10] C. C. Chang, "A new proof of the completeness of the Łukasiewicz axioms," Transactions of the American Mathematical Society, vol. 93, pp. 74-80, 1959.

[11] R. L. O. Cignoli, I. M. L. D’Ottaviano, and D. Mundici, Algebraic Foundations of Many-Valued Reasoning, vol. 7 of Trends in Logic-Studia Logica Library, Kluwer Academic Publishers, Dordrecht, The Netherlands, 2000. 


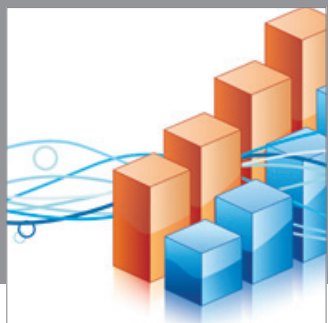

Advances in

Operations Research

mansans

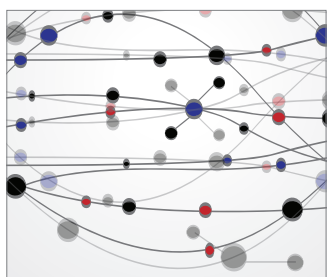

The Scientific World Journal
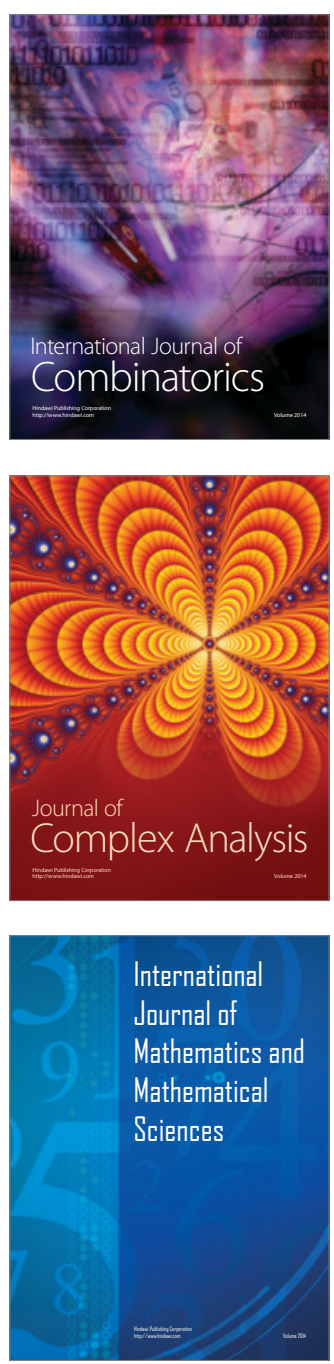
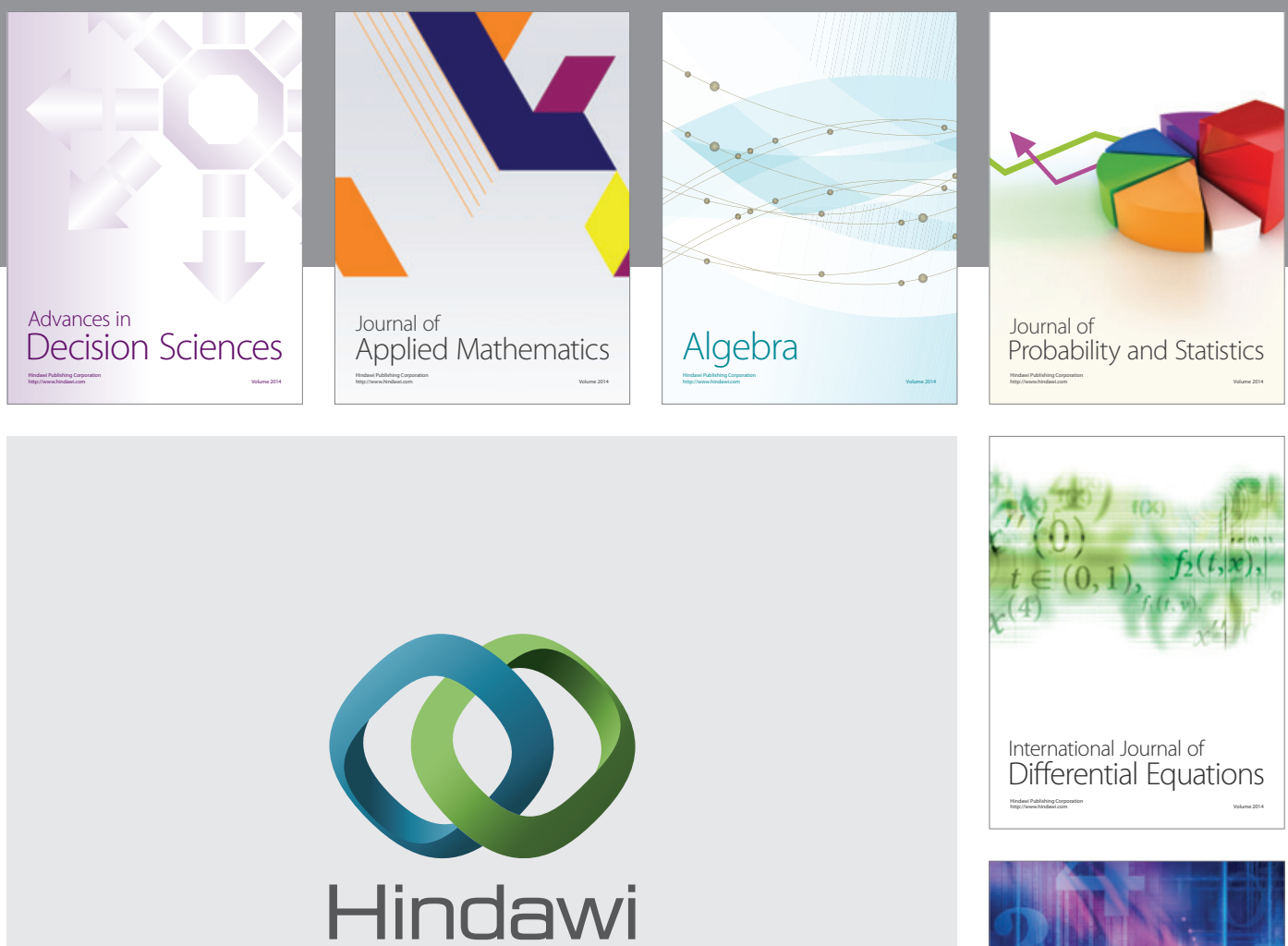

Submit your manuscripts at http://www.hindawi.com
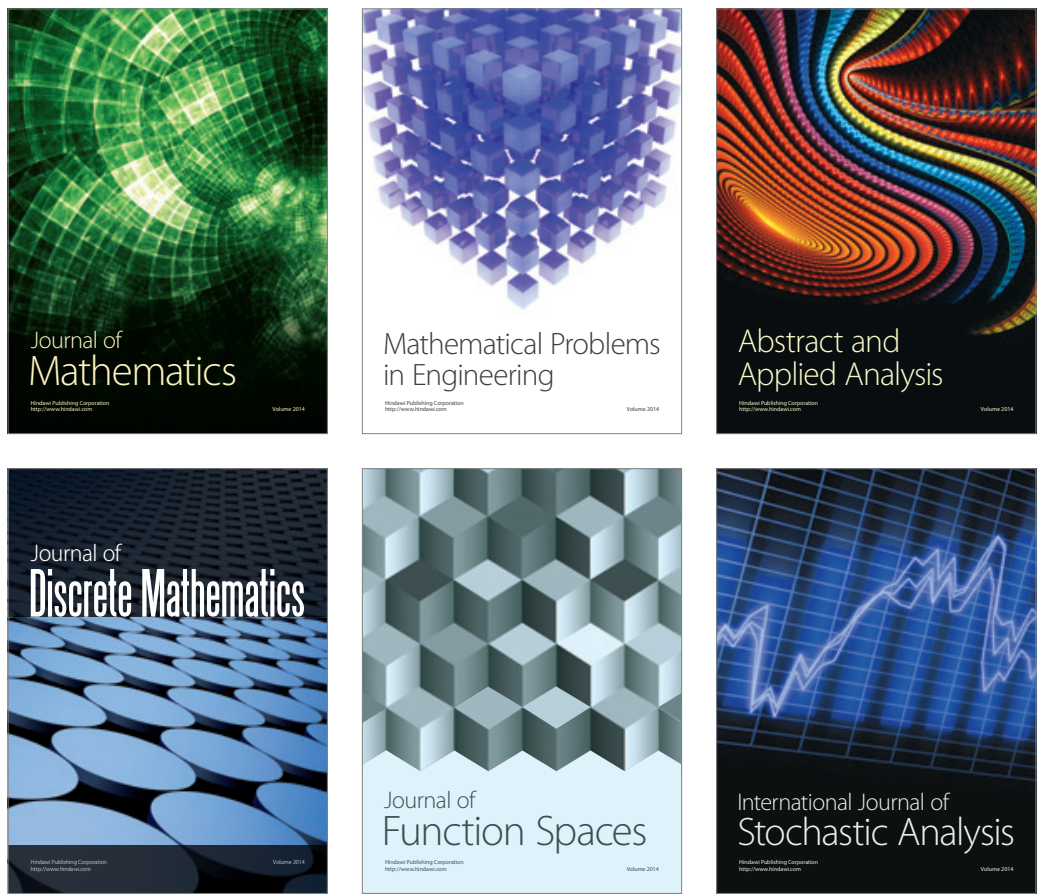

Journal of

Function Spaces

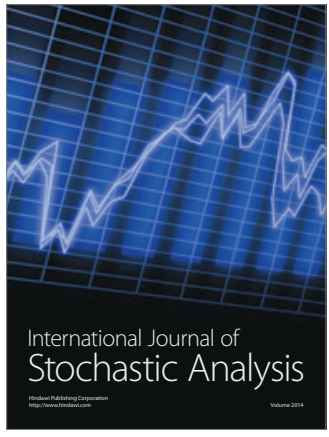

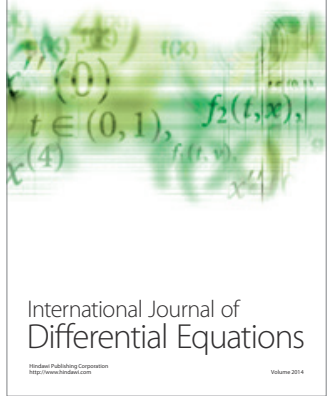
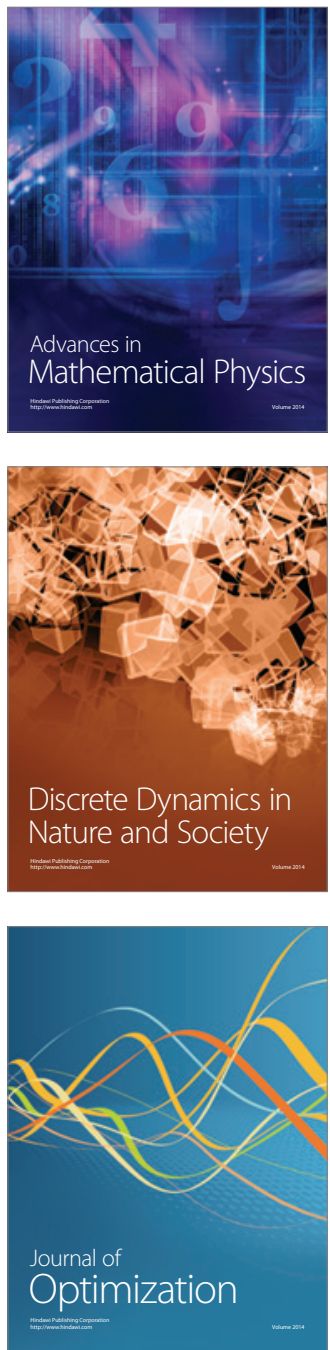